\title{
ORTHOLOGIC DESARGUES' FIGURE
}

\author{
SAHIB RAM MANDAN \\ (Received 5 October 1977; revised 9 April 1979) \\ Communicated by J. B. Miller
}

\begin{abstract}
In 1894 Sondat published a theorem that the centre of perspectivity and the 2 orthologic centres of any 2 bilogic (perspective as well as orthologic) triangles lie on a line perpendicular to their axis of perspectivity. Thébault (1952) gave an elementary proof of this theorem. Here we give two new proofs, one synthetic and the other analytic, and then deduce the existence of an orthologic Desargues' figure where all the 10 pairs of perspective triangles in it are orthologic. Consequently we arrive at an orthologic Veronese configuration of 15 points and 10 pairs of perpendicular lines studied in 5 different ways.

We also give a construction for special pairs of bilogic triangles whose vertices lie on 3 lines perpendicular to their common axis of perspectivity.
\end{abstract}

1980 Mathematics subject classification (Amer. Math. Soc.): 51 A 20.

\section{Introduction}

A Desargues' figure $f$ occurs as the section by the plane $p$ of perspectivity of a special pair of bilogic tetrahedra $(X)=X_{1} X_{2} X_{3} X_{4}(X=A, B)$ such that the joins $p_{i}=A_{i} B_{i}$ of their corresponding vertices are perpendicular to $p$ (Mandan (1979)) at the points $0 i(=i 0)$, their corresponding edges $X_{i} X_{j}$ meet $p$ in the same 6 points $i j(=j i)$ and corresponding faces $X_{i} X_{j} X_{k}$ in the same 4 lines $i j k$ $(i, j, k=1,2,3,4)$. The 2 triads of points

$$
T=(01,02,03), \quad T^{\prime}=(14,24,34)
$$

form a pair of triangles perspective from the point 04 and its corresponding line 123 in $f$. It is easy to construct such a pair to be orthologic such that perpendiculars from the vertices of $T$ to the corresponding sides of $T^{\prime}$ concur at a point 05 (say) and then (Mandan (1965), p. 49) those from the vertices of $T^{\prime}$ to the corresponding sides of $T$ concur at a point 45 (say). $T, T^{\prime}$ are then said to form a bilogic (Thébault (1952), p. 25; (1955), p. 67) pair with 05,45 as their orthologic centres (o.c.) as 
shown in Figure 1 where the symbol for any line is the union of the symbols of any 2 points on it and dually that for any point is the intersection of those of any 2 lines on it.

\section{New proofs of Sondat's theorem}

The theorem before us now is to prove that the perpendicular from the centre of perspectivity 04 of the 2 triangles $T, T^{\prime}$ to their axis of perspectivity 123 passes through their 2 o.c.: 05,45 .

Proof I. It is known (Court (1964), p. 73, Ex. 3) that if the 3 altitudes of a tetrahedron $(C)=C_{1} C_{2} C_{3} C_{4}$ from its 3 vertices $C_{1}, C_{2}, C_{3}$ concur at a point $C$, its 4 th altitude also passes through $C_{5}$ to make it orthocentric.

Let $0 i$ be the orthogonal projections of the 5 points $C_{i}$ and $0 i j$ of the 10 lines $C_{i} C_{j}$ in a plane $p$ that meets these lines in 10 points $i j$ and the planes $C_{i} C_{j} C_{k}$ in 10 lines $i j k(i, j, k=1,2,3,4,5)$. The concurency of the 3 altitudes of $(C)$ implies the perpendicularity of the 3 lines: $015,025,035$ to the 3 lines: 234, 134, 124 respectively such that the 2 triangles: $T=(01,02,03), T^{\prime}=(14,24,34)$ perspective from 04 and 123 form the Desargues' figure $f$ and are orthologic with 05 as an orthologic centre. The passing of the 4 th altitude of $(C)$ through $C_{5}$ implies the perpendicularity of the 3 lines: $145,245,345$ to the 3 lines: $023,013,012$ respectively as well as that of the line 045 to the line 123 such that 45 is the second orthologic centre of $T, T^{\prime}$ and the 3 points: $04,05,45$ lie on the line 045 as desired to establish Sondat's theorem.

Proof II. We may define perpendicular lines as those which are conjugate for a conic $q$, called absolute (Baker (1930), p. 63), that may reduce to the two circular points at infinity. Let

$$
Q=\left\|\begin{array}{lll}
a & h & g \\
h & b & f \\
g & f & c
\end{array}\right\|
$$

be the matrix of the tangential equation of $q, T$ the triangle of reference and 04 the unit point $(1,1,1)$. The vertices of $T^{\prime}$ may then be taken as

$$
14=(x, 1,1), \quad 24=(1, y, 1), \quad 34=(1,1, z)
$$

for some numbers $x, y, z \neq 1$. Since $12=(1-x,-(1-y), 0)$, and so on, the coordinate-vector of the line 123 is seen to be

$$
[123]=[1 /(1-x), 1 /(1-y), 1 /(1-z)] .
$$


The coordinates of the poles for $q$ of the sides of $T$ form the rows of $Q$, and then the lines on the vertices of $T^{\prime}$ conjugate for $q$ (perpendicular) to the corresponding sides of $T$ have their coordinate-vectors derived from the matrices

as

$$
\left\|\begin{array}{lll}
x & 1 & 1 \\
a & h & g
\end{array}\right\|,\left\|\begin{array}{lll}
1 & y & 1 \\
h & b & f
\end{array}\right\|,\left\|\begin{array}{lll}
1 & 1 & z \\
g & f & c
\end{array}\right\|
$$

$$
\begin{aligned}
& L_{1}=[g-h, a-x g, x h-a], \quad L_{2}=[y f-b, h-f, b-y h], \\
& L_{3}=[c-z f, z g-c, f-g] .
\end{aligned}
$$

$T, T^{\prime}$ will be orthologic if the last three lines concur at a point 45 (say) so that any line whose coordinate-vector is a linear combination of $L_{1}, L_{2}, L_{3}$ will pass through 45.

Now the coordinate-vector of the pole of the line 123 for $q$ is given by the product [123] $Q$ so that the coordinate-vector of the line on the point 04 conjugate for $q$ (perpendicular) to 123 is found from the matrix

$$
\left\|\frac{a}{1-x}+\frac{h}{1-y}+\frac{g}{1-z} \frac{h}{1-x}+\frac{b}{1-y}+\frac{f}{1-z} \frac{g}{1-x}+\frac{1}{1-y}+\frac{c}{1-z}\right\|
$$

to be $L_{1} /(1-x)+L_{2} /(1-y)+L_{3} /(1-z)$ which shows that it lies on 45 , and similarly on 05 also as desired.

\section{Orthologic character of the figure $f$}

Let us first enumerate all the 9 pairs of perspective triangles in the Desargues' figure $f$ other than $T, T^{\prime}$ as follows:

(i) $(04,24,34),(01,12,31)$ perspective from 14 and 023 ,

(ii) $(04,14,34),(02,12,23)$ perspective from 24 and 013 ,

(iii) $(04,14,24),(03,13,23)$ perspective from 34 and 012 ,

(iv) $(02,03,04),(12,13,14)$ perspective from 01 and 234 ,

(v) $(01,03,04),(12,23,24)$ perspective from 02 and 134 ,

(vi) $(01,02,04),(13,23,34)$ perspective from 03 and 124 ,

(vii) $(02,12,24),(03,13,34)$ perspective from 23 and 014 ,

(viii) $(03,23,34),(01,12,14)$ perspective from 31 and 024 ,

(ix) $(01,13,14),(02,23,24)$ perspective from 12 and 034.

The first 6 pairs are seen to be orthologic, with 45 as an orthologic centre for the first 3 pairs and 05 for the next 3 pairs, as evident from the concurrence of the perpendiculars from the vertices of the first triangle to the corresponding sides of the second in each pair. The perpendiculars from the vertices of the second triangles to the corresponding sides of the first in the first 6 pairs then (Gerber 
(1975), p. 50) concur at their second o.c. which may be named as 15, 25, 35 respectively for the first 3 pairs and are seen to be the same for the next 3 pairs respectively by Sondat's theorem. Consequently the last 3 pairs also become orthologic with 25,$35 ; 35,15 ; 15,25$ as the pairs of their o.c. respectively.

Thus the figure $f$ has 5 o.c.: $05,15,25,35,45$.

\section{Orthologic Veronese configuration}

It is now easy to count the total number of points and lines in this figure as 15 and 20 respectively to form a Veronese configuration (V.C.) such that 4 lines: $i j k, i j u, i j v, i j w$ pass through each point $i j$, and 3 points: $i j, j k, k i$ lie on each line iik perpendicular to its companion line $u v w(i, j, k, u, v, w=0,1,2,3,4,5)$ as shown in Figure 1.

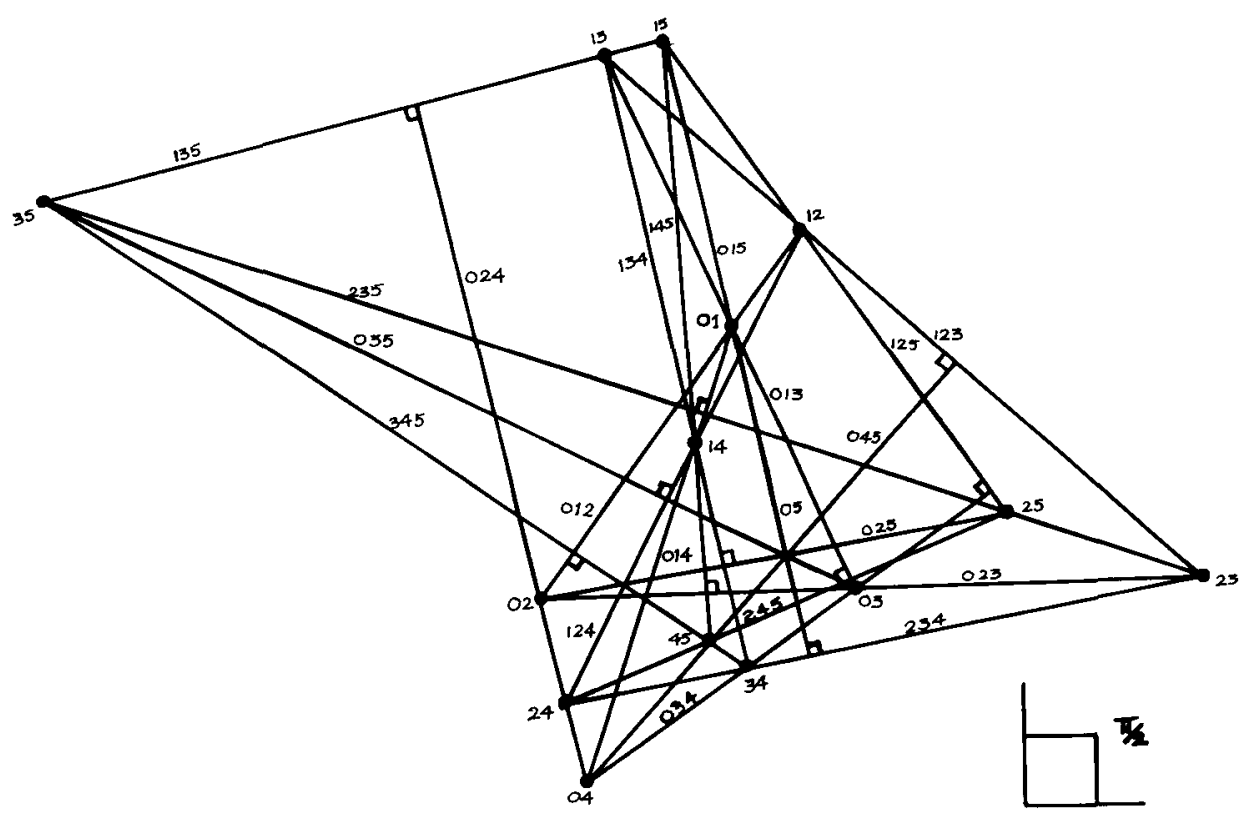

Figure 1. An orthologic Veronese configuration.

Such a figure, its specialization and dual occur already in the literature (see Baker (1929), frontispiece; (1930), pp. 214-218; (1934), p. 34; Court (1954), p. 15; Mandan (1960), p. 36; (1966), p. 15; Salmon (1964), p. 382; Veblen and Young (1938), p. 42) but without the orthologic character which is our specialization here. It may be studied in 5 different ways as follows:

(i) Any Desargues' figure $10_{3}$ (Coxeter (1969), p. 238; Pedoe (1963), p. 25; 
Room (1967), p. 24) may be regarded, in 5 ways, as a quadrilateral $j k u v$ (formed of 4 lines: $j k u, k u v, u v j, v j k)$ and a quadrangle $(i j, i k, i u, i v)$, so interwoven that the 6 vertices of the former lie on the 6 sides of the latter (Baker (1930), p. 251; Coxeter (1975), p. 231). When it is orthologic, the perpendiculars from the vertices of the quadrangle to the corresponding sides of the quadrilateral in the figure concur at one of its 5 o.c.: $i w, j w, k w, u w, v w$ which form a complete 5-point (Veblen and Young (1938), p. 38) whose 10 sides pass through its 10 points and are perpendicular to their corresponding lines in it. Such an orthologic Desargues' figure may then be justifiably named as $(w)$ such that our V.C. contains 6 such figures: (0), (1), (2), (3), (4), (5) =f.

(ii) Any 2 quandrangles are said to be orthologic (Gerber (1977), p. 54; Mandan (1977b), p. 414) if each side of one is perpendicular to a side of the other such that every one of the 4 pairs of their corresponding triangles are orthologic with their remainding 2 vertices as o.c.

It is interesting to observe that every point $i j$ of our V.C. is the centre of perspectivity of 2 bilogic quadrangles: ( $i k, i u, i v, i w),(j k, j u, j v, j w)$ leaving behind its 6 points as the meets of their corresponding sides to form vertices of their quadrilateral of perspectivity kuvw whose sides are the axes of perspectivity of their corresponding triangles perpendicular to the joins of their remaining vertices respectively.

(iii) Further we notice that every line $i j k$ of our V.C. is the common axis of

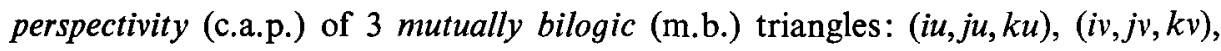
$(i w, j w, k w)$ whose centres of perspectivity (c.p.): $u w, v w, w u$ are also their o.c. on its common line $u v w$ that happens to be the c.a.p. of another triad of m.b. triangles:

$(i u, i v, i w),(j u, j v, j w),(k u, k v, k w)$ with $i j, j k, k i$ as their c.p. as well as o.c. on $i j k$.

(iv) It leads to the construction (Mandan (1979)) of a special pair of bilogic tetrahedra $(X)=X_{k} X_{u} X_{v} X_{w}(X=A, B)$ with joins of their corresponding vertices perpendicular to the plane $p$ of our V.C. at the vertices of one of the 2 bilogic quadrangles in (ii) and their corresponding edges passing through the vertices of the quadrilateral $k u v w$, the vertices of the other quadrangle being the common meets of $p$ with the perpendiculars from the vertices of each tetrahedron to the corresponding faces of the other and the centre of perspectivity $i j$ of the quadrangles the common orthogonal projection in $p$ of the 2 o.c. of $(X)$.

(v) In 1975 Gerber (private communication) conjectured that if the perpendiculars from the orthogonal projections in a plane $p$ of the vertices of a tetrahedron to the faces of another in a correspondence concur, those from the orthogonal projections in $p$ of the vertices of the second to the corresponding faces of the first also concur. It is shown (Mandan (1977b), p. 414) that it happens only if the 
orthogonal projections in $p$ of the vertices of the 2 tetrahedra form 2 orthologic quadrangles.

We now have 15 pairs of bilogic quadrangles in our V.C. that amount to 15 ways to construct pairs of Gerber tetrahedra (satisfying the conditions of Gerber's conjecture) as illustrated below for one such pair.

Draw secants from any point $P_{i j}$, on the perpendicular to $p$ at the centre of perspectivity $i j$ of the 2 bilogic quadrangles in (ii), to those at the pairs of their corresponding vertices to obtain the pairs of corresponding vertices of 2 Gerber tetrahedra perspective from $P_{i j}$ such that the orthogonal projections of the 6 meets of their corresponding edges in $p$ are the remaining 6 points of our V.C.

The 15 points of our V.C. are thus the orthogonal projections in $p$ of the 15 points of the figure of any pair of perspective Gerber tetrahedra.

\section{Special pairs of bilogic triangles}

Taking the plane $p$ in the first proof of Sondat's theorem parallel to an edge, say $C_{1} C_{2}$, of the orthocentric tetrahedron $(C)$ there, we obtain a special pair of bilogic triangles: $(13,14,15),(23,24,25)$ as shown in Figure 2 . They are perspective from the point 12 at infinity, the joins of their corresponding vertices are

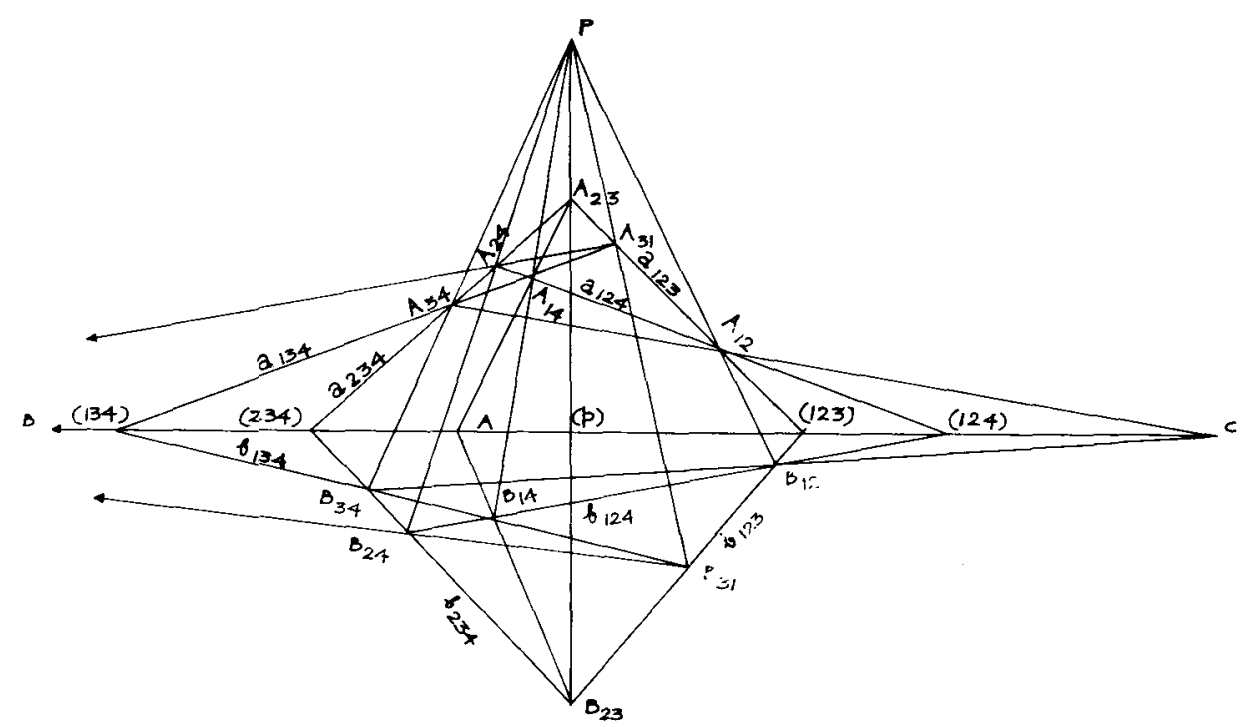

FIGURE 2. A special pair of biologic triangles: $(13,14,15),(23,24,25)$. 
perpendicular to their axis of perspectivity 345 (Mandan (1977a), p. 57), and the points 01,02 are their o.c. such that the quadrilateral 0345 in our V.C. now collapses into a line as the meet 345 of $p$ with the plane $C_{3} C_{4} C_{5}$ of the 2 altitudes $C_{3} C_{5}, C_{4} C_{5}$ of $(C)$ perpendicular to its edge $C_{1} C_{2}$ and therefore to $p$ to make the orthogonal projections $0 i(i=3,4,5)$ in $p$ of the 3 points $C_{i}$ lie on 345 .

\section{Acknowledgements}

I take this opportunity to acknowledge gratefully the gift of a reprint of a paper of Thébault (1952) presented to me by the referee besides his valuable suggestions to improve the quality of this paper. My thanks are also due to Mr Satya Prakash Sharma (Manager, Central Design Office, Garden Reach Shipbuilders and Engineers, Calcutta) and an architecture student G. Mira for drawing the figures.

\section{References}

H. F. Baker (1929), Principies of geometry I (Cambridge University Press).

H. F. Baker (1930), Principles of geometry II (Cambridge University Press).

H. F. Baker (1934), Principles of geometry III (Cambridge University Press).

N. A. Court (1954), 'Desargues and his strange theorem', Scripta Math. 20, 1-20.

N. A. Court (1964), Modern pure solid geometry (Chelsea).

H. S. M. Coxeter (1964), Projective geometry (Blaisdell, New York).

H. S. M. Coxeter (1969), Introduction to geometry (Wiley, New York).

H. S. M. Coxeter (1975), 'Desargues' configurations and their collineation groups', Math. Proc. Cambridge Philos. Soc. 78, 227-246.

L. Gerber (1975), 'Associated and perspective simplexes', Trans. Amer. Math. Soc. 201, 43-55.

L. Gerber (1977), 'Associated and skew-orthologic simplexes', Trans. Amer. Math. Soc. 231, 47-63.

S. R. Mandan (1960), 'A configuration of 600 lines in [4]', Rev. Fac. Sci. Univ. Istanbul A25, $17-44$.

S. R. Mandan (1965), 'Concurrent lines related to 2 plane triangles', J. Sci. Engrg. Res. 9, 47-50.

S. R. Mandan (1966), 'Perspective simplexes', J. Austral. Math. Soc. 6, 11-17.

S. R. Mandan (1977a), 'Skew orthologic perspective simplexes', J. Mathematical and Physical Sci. 11, 569-576.

S. R. Mandan (1977b), 'On a Gerber's conjecture', Atti Accad. Naz. Lincei Rend. Cl. Sci. Fis. Mat. Natur. (Ser. VIII) 61, 411-419.

S. R. Mandan (1979), 'Special pairs of semi-bilogic and bilogic tetrahedra', J. Austral. Math. Soc. (Series A) 28, 303-308.

D. Pedoe (1963), Introduction to projective geometry (Pergamon, Oxford).

T. G. Room (1967), A background to geometry (Cambridge University Press).

G. Salmon (1964), Conic Sections (Chelsea).

P. Sondat (1894), 'Question 38', L'intermédiaire des mathématiciens 1, 10 (solved by Sollerstinsky, L'intermédiaire des mathématiciens 1, 44-45).

V. Thébault (1952), 'Perspective and orthologic triangles and tetrahedrons', Amer. Math. Monthly $59,24-28$. 
V. Thébault (1955), Parmi les belles figures de la géométrie dans l'espace (Libraire Vuibert, Paris).

O. Veblen and J. W. Young (1938), Projective geometry I (Ginn, New York).

Flat 19, "Vitaya", Chhedanagar, Bombay-400089

India 